\title{
Chitosan-g-polyaniline: a creatine amidinohydrolase immobilization matrix for creatine biosensor
}

\author{
A. Tiwari ${ }^{1 *}$, S. K. Shukla \\ ${ }^{1}$ Division of Engineering Materials, National Physical Laboratory, Dr. K.S. Krishnan Marg, New Delhi-110 012, India \\ (Present address: Department of Mechanical Engineering, University of Wisconsin-Milwaukee, Milwaukee, WI 53211, \\ USA) \\ 2Department of Polymer Science, Bhaskaracharya College of Applied Sciences, University of Delhi, New Delhi-110 075, \\ India
}

Received 7 May 2009; accepted in revised form 13 June 2009

\begin{abstract}
A novel matrix composed of chitosan-graft-polyaniline (CHIT-g-PANI) was electrochemically prepared to investigate the immobilization of creatine amidinohydrolase (CAH). CAH enzyme was covalently immobilized with the CHIT- $g$-PANI matrix using glutaraldehyde as a linker. The resulting CAH/CHIT- $g$-PANI biomatrix was characterized with Fourier transform infrared spectroscopy (FTIR), atomic force microscopy (AFM), contact angle measurement and cyclic voltammetry (CV) taking CHIT-g-PANI as a reference. The influence of various parameters on CAH enzyme activity within the matrix was investigated including $\mathrm{pH}$, temperature, and time. The Michaelis-Menten constant and apparent activities for the CAH enzyme were calculated to be $0.51 \mathrm{mM}$ and $83.59 \mathrm{mg} / \mathrm{cm}^{2}$, respectively; indicating CHIT- $g$-PANI matrix has a high affinity to immobilize CAH enzyme.
\end{abstract}

Keywords: polymer synthesis, molecular engineering, chitosan-graft-polyaniline, creatine amidinohydrolase, immobilization matrix

\section{Introduction}

Creatinine is an important clinical analyte for the diagnosis of renal and muscular dysfunction [1]. It is a dehydrogenated form of creatine (i.e., a metabolic byproduct of amino acid) that provides energy to muscles tissue. The normal clinical range of creatinine in the human blood is ranging from 44 to $106 \mu \mathrm{M}$; however, it can exceed up to $1000 \mu \mathrm{M}$ during nephrons malfunction [2]. Therefore, precise monitoring of creatinine in the blood is compulsory during routine check up.

Most of the existing creatinine biosensors utilize creatine amidinohydrolase $(\mathrm{CAH})$ as a sensing element [3-5]. Typically, CAH is a homodimer enzyme, which hydrolyzes creatinine into urea and sarcosine [6]. The concentration of creatinine is quantitatively measured by monitoring the librated hydrolyzed byproducts using a range of transducers such as amperometric, potentiometric, optical, etc. $[7,8]$. However, these biosensors are offered only limited biosensor stability due to low functional stability of CAH enzyme within the matrix [9]. In practice, performance of enzyme based biosensors usually depends on the physicochemical properties of the electrode materials as well as process of the enzyme immobilization and also enzyme concentration on the electrode surface [10]. Although, various matrices are reported in the literature for the immobilization of CAH enzyme to use in creatinine biosensors, the method of immobilization and electrode matrices, both are considered promising factor during the determination of the operational and

*Corresponding author, e-mail: tiwari@uwm.edu

(c) BME-PT 
storage stability of the biosensors [11]. The low intrinsic stability of CAH enzyme has encouraged for applying biomaterials engineering to improving stability [12]. Specifically, this study investigates the feasibility of CHIT- $g$-PANI as potential CAH immobilization matrix.

Polyaniline (PANI) is a unique conducting polymer and its reversible conductivity can be controlled by the protonation of the imine sites or the oxidation of the main polymer chain [13]. However, traditional PANI does not have such enzyme loading capability as its chemically modified forms [14]. Meanwhile, Chitosan (CHIT) has become a widespread biopolymer owing to its remarkable chemical and biological characteristics. It is a hydrophilic material due to the presence of both amino and hydroxyl groups; however, CHIT is insoluble in water and aqueous basic media [15]. It was chosen as the orientation directing matrix because there are large quantities of amino and hydroxyl groups on the CHIT units, which have a strong binding ability to enzyme and DNA $[14,16]$. Moreover, in acidic condition CHIT showed a cationic nature that may provide an electrostatic core environment to zwitterion molecules such as enzyme.

In the present work, we describe the fabrication of a novel matrix based on CHIT- $g$-PANI, covalent immobilization and stabilization conditions for the $\mathrm{CAH}$ enzyme. Further, Michaelis-Menten kinetic parameter and apparent activities for the $\mathrm{CAH}$ enzyme were calculated correspondingly using electrochemical and photometric techniques.

\section{Experimental}

\subsection{Materials}

Chitosan (CHIT, $>85 \%$ deacetylated, $M_{w} 1.86 \cdot 10^{5}$ ), aniline (99\%), creatinine (anhydrous) and urease (Urs, from Canavalia ensiformis) were purchased from Sigma-Aldrich, USA. Creatine amidinohy- drolase (CAH, from Actinobacillus sp., CRH-211) was taken from Toyobo Co., Ltd. and used without further purification. All supplementary chemicals were of analytical grades and solutions were prepared with nanopure water. Indium-tin-oxide (ITO) coated glass sheets (Balzers) with a resistance of $15 \Omega / \mathrm{cm}^{2}$ were used as substrates for the deposition of electrodes.

\subsection{Preparation of CHIT solution}

CHIT solution was prepared by dissolving $2.0 \mathrm{~g}$ of CHIT flakes into $100 \mathrm{ml}$ of $1.0 \%$ acetic acid and stirred for three hours at room temperature until completely dissolved. The CHIT solution was stored in a refrigerator when not in use.

\subsection{Electrochemical synthesis of CHIT-g-PANI}

Aniline $(180 \mu \mathrm{l})$, and $2.0 \%$ CHIT solution $(75 \mu \mathrm{l})$ was mixed with $10 \mathrm{ml}$ of $0.5 \mathrm{M} \mathrm{HCl}$ in an electrochemical cell and the mixture was ultrasonically agitated for about four hours. The CHIT- $g$-PANI was chronoamperometrically synthesized onto an ITO coated glass surface using a three-electrode assembly with ITO glass as working, platinum as counter, and $\mathrm{Ag} / \mathrm{AgCl}$ as reference electrodes at a potential of $0.9 \mathrm{~V}$ and duration of 200 seconds. The resulting CHIT- $g$-PANI/ITO electrode was washed with deionized water followed by a phosphate buffer saline (PBS) solution of $\mathrm{pH} 7.0$ in order to neutralize the electrode surface.

\subsection{Immobilization of CAH enzyme}

The CAH enzyme was covalently immobilized over the CHIT-g-PANI matrix using glutaraldehyde as a linker. Ten $\mu 1$ of $25 \%$ gluteraldehyde solution was spread over the CHIT-g-PANI/ITO
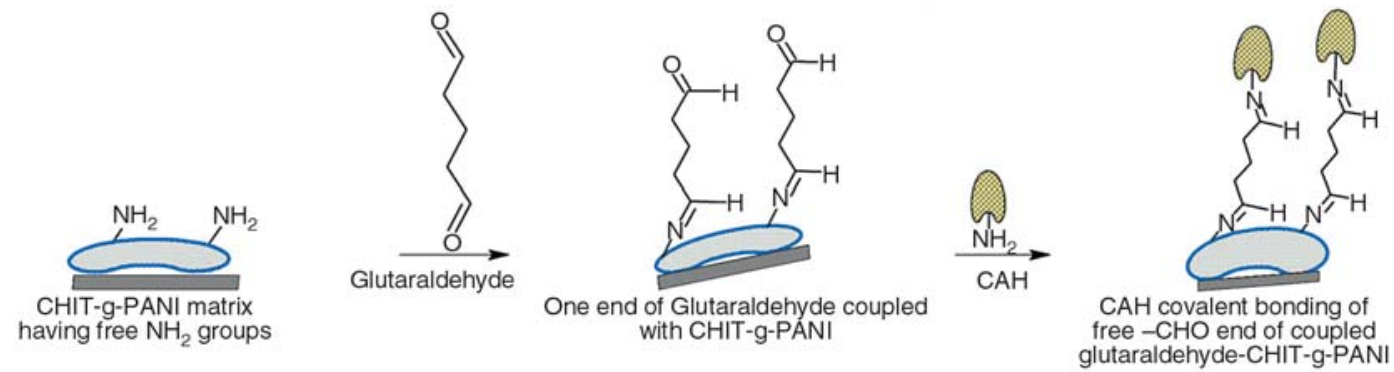

Figure 1. Covalent immobilization of the CAH on CHIT-g-PANI graft copolymer matrix using gluteraldehyde as a linker 
electrode and kept for five hours at room temperature, then $10 \mu \mathrm{l} \mathrm{CAH} \mathrm{(350} \mathrm{units/} \mu \mathrm{l})$ was spread on top of the electrode and dried (Figure 1). The resulting CAH/CHIT- $g$-PANI/ITO bioelectrode was thoroughly washed with PBS of $\mathrm{pH} 7.0$ to rinse off any loosely bound CAH enzyme from the bioelectrode.

\subsection{Characterization}

CHIT- $g$-PANI and CAH/CHIT- $g$-PANI biomatrix were characterized using FTIR, AFM, contact angle, and CV measurements. FTIR spectra were recorded on a Perkin Elmer, Spectrum BX II spectrophotometer. The surface topology of the electrodes was studied using AFM (Veeco DICP2) under the tapping mode. Contact angles of the electrodes were measured with a video-based, automatic research grade contact angle measurement system, FDSC-OCA 20 using water as liquid phase.

Electrochemical measurements of the electrodes were carried out on a Potentiostat/Glavanostat (Princeton Applied Research, 273A) unit with three electrodes in a $50 \mathrm{mM}$ phosphate buffer solution $(\mathrm{pH} 7.0,0.9 \% \mathrm{NaCl})$ containing $5 \mathrm{mM} \mathrm{Fe}(\mathrm{CN})_{6}^{3-14}$. The working electrode was either CHIT- $g$-PANI/ ITO or CAH/CHIT- $g$-PANI/ITO. Platinum foil and $\mathrm{Ag} / \mathrm{AgCl}$ were used as the counter and reference electrodes, respectively. All measurements were carried out at $25^{\circ} \mathrm{C}$.

\subsection{Photometric apparent enzyme activity measurement}

Photometric study was performed using a Varian Cary 100 Bio UV-visible spectrophotometer. For photometric measurements, a CAH/CHIT- $g$-PANI/ ITO bioelectrode was dipped in a $5 \mathrm{ml}$ phosphate buffer solution $(50 \mathrm{mM}, \mathrm{pH} 7.0)$, which contained $200 \mu \mathrm{l}$ of Nessler's solution, $50 \mu \mathrm{l}$ urease enzyme $(50 \mathrm{mg} / \mathrm{ml})$ and $1 \mathrm{ml}$ of creatinine solution with varying concentrations. After $3 \mathrm{~min}$ of CAH/CHIT$g$-PANI/ITO electrode incubation, the absorbance of the colored product (i.e., $\mathrm{NH}_{2} \mathrm{Hg}_{2} \mathrm{I}_{3}$, a complex formed between the Nessler's reagent and ammonia produced by the enzymatic hydrolysis of creatinine) in the solution at $\lambda_{\max } 385 \mathrm{~nm}$, was measured to monitor the CAH enzyme kinetics.

\section{Results and discussion}

\subsection{Graft copolymerization, enzyme immobilization and characterization}

In the electrochemical copolymerization synthesis, the aniline monomer initially became protonated with $\mathrm{HCl}$ and propagated to form an intermediate called PANI radical cation (Figure 2a) [17-19].

PANI radical cation simultaneously generated CHIT macro radicals (Figure $2 \mathrm{~b}$ ) by the abstraction of hydrogen from the $-\mathrm{OH}$ and $-\mathrm{NH}_{2}$ groups of the CHIT macromolecules $[15,16]$.

The PANI cation radicals and CHIT macro radicals then copolymerized and yielded CHIT- $g$-PANI (Figure 2c).

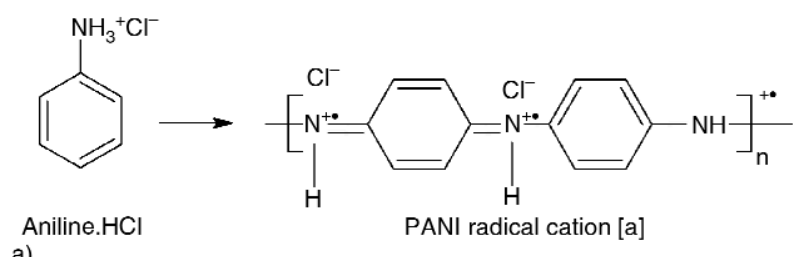

a)

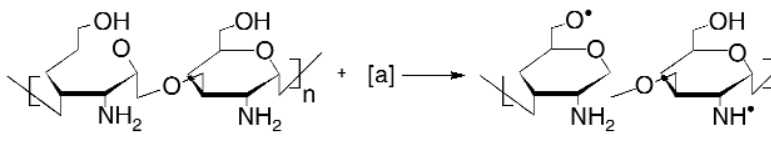

b)

b)

$$
\text { CHIT macroradical }[\mathrm{b}]
$$

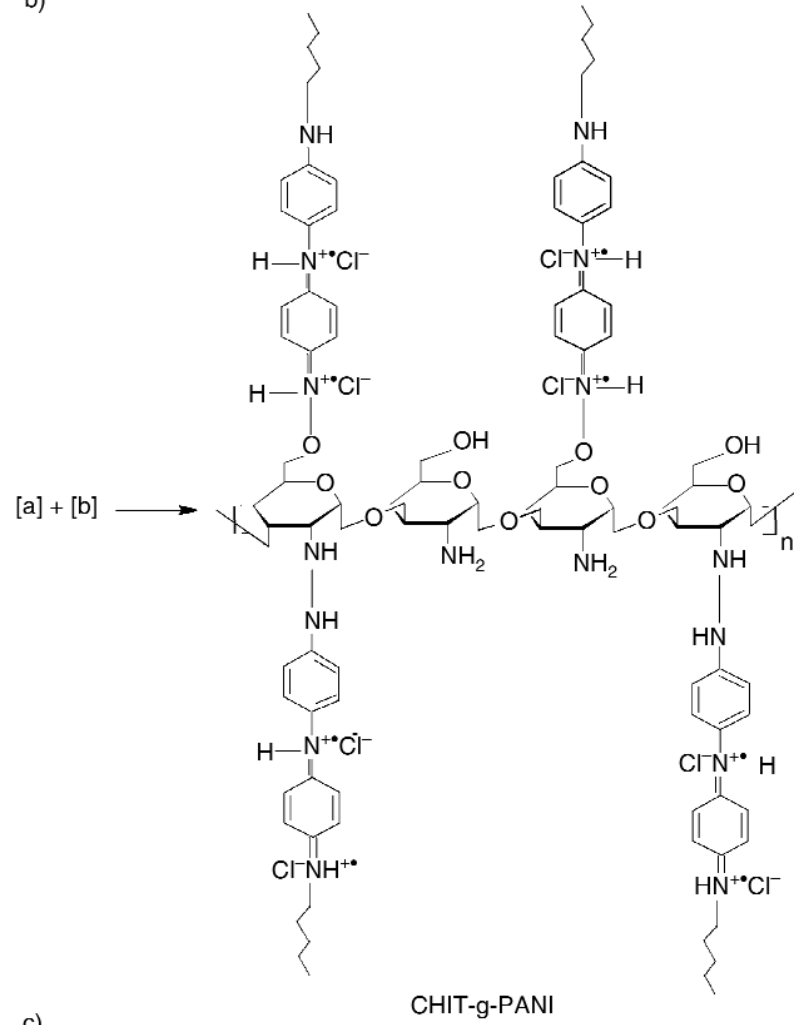

c)

Figure 2. Electrochemical copolymerization synthesis. a) PANI radical cation, b) CHIT macro radical, c) CHIT-g-PANI 


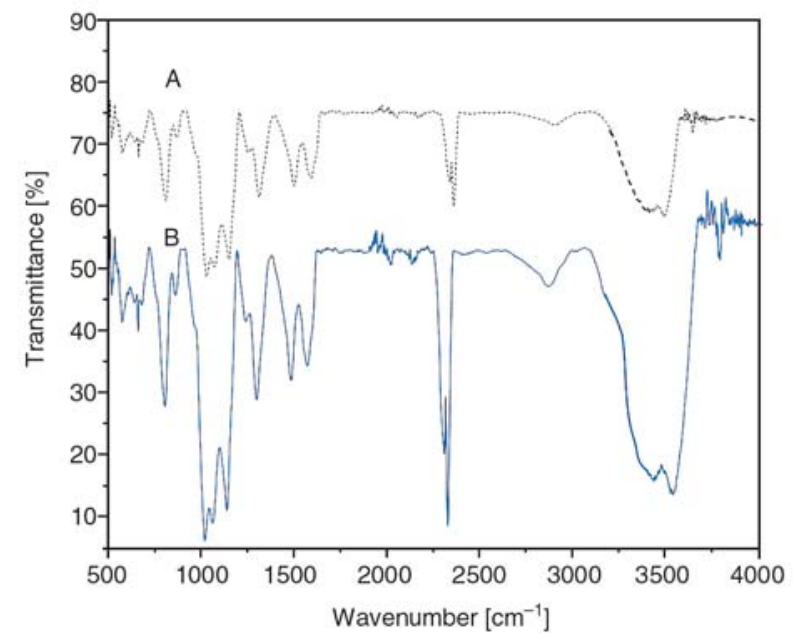

Figure 3. FTIR spectra of the (spectrum A) CHIT- $g$-PANI and (spectrum B) CAH/CHIT- $g$-PANI

The FTIR spectrum of the CHIT- $g$-PANI/ITO electrode (Figure 3, spectrum A) illustrated the characteristic peaks of PANI, as well as CHIT [16]. The following key characteristic bands were observed: 1) 3150 to $3542 \mathrm{~cm}^{-1}$ (free O-H stretching and $\mathrm{N}-\mathrm{H}$ stretching with hydrogen bonded secondary amino groups); 2) $3021 \mathrm{~cm}^{-1}$ (aromatic C-H stretching); 2926 and $2862 \mathrm{~cm}^{-1}$ (aliphatic $\mathrm{C}-\mathrm{H}$ stretching); 3) $1632 \mathrm{~cm}^{-1}(\mathrm{C}=\mathrm{O}$ stretching of carbonyl group, typical saccharide absorption); 4) $1584 \mathrm{~cm}^{-1}$ $\left(\mathrm{C}=\mathrm{C}\right.$ stretching of quinoid rings); 5) $1483 \mathrm{~cm}^{-1}$ $(\mathrm{C}=\mathrm{C}$ stretching vibration of benzenoid rings); and 6) $1245 \mathrm{~cm}^{-1}$ (C-N stretching). The absorption band of the $\mathrm{N}=\mathrm{Q}=\mathrm{N}$ bending vibration of protonated pure PANI was observed at $1236 \mathrm{~cm}^{-1}$, but shifted to $1123 \mathrm{~cm}^{-1}$ in the CHIT- $g$-PANI copolymer due to the steric effect of CHIT [16].

Stable enzyme-substrate coupling was achieved with gluteraldehyde as a cross-linking agent [20]. One end of the gluteraldehyde is attached to the $-\mathrm{NH}_{2}$ group of the CHIT- $g$-PANI/ITO electrode through a reaction between the $-\mathrm{CHO}$ end group of glutaraldehyde and the $-\mathrm{NH}_{2}$ groups of terminal PANI and CHIT. The other end of the gluteraldehyde is attached to $\mathrm{CAH}$ through a reaction between the - $\mathrm{CHO}$ group of gluteraldehyde and $-\mathrm{NH}_{2}$ group of $\mathrm{CAH}$, which resulted in a CAH/ CHIT- $g$-PANI/ITO bioelectrode (Figure 1). It may be possible that some of the glutaraldehyde attached within the free $-\mathrm{NH}_{2}$ groups of the CHIT- $g$-PANI matrix but probability of cross linking between $-\mathrm{NH}_{2}$ functionalized electrode to enzyme is quite common [20]. The FTIR spectrum of the CAH/

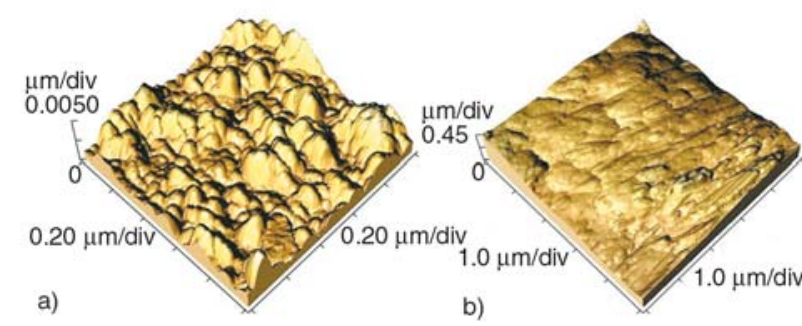

Figure 4. AFM images of the (a) CHIT- $g$-PANI and (b) CAH/CHIT- $g$-PANI

CHIT- $g$-PANI/ITO electrode (Figure 3, spectrum B) showed phosphate vibration at $1224 \mathrm{~cm}^{-1}$ (accredited from phosphate backbone of immobilized cDNA) with the peaks broadening at 1) 3123 to $3564 \mathrm{~cm}^{-1}$ (addition of $\mathrm{N}-\mathrm{H}$ stretching vibration); 2) 3022 to $2856 \mathrm{~cm}^{-1}$; and 3) $1631 \mathrm{~cm}^{-1}$ to 1664 due to the attachment of CAH with the CHIT- $g$ PANI matrix. Hence, FTIR spectra confirmed the immobilization of CAH onto the CHIT-gPANI/ITO electrode.

The surface morphology of the electrodes was observed with AFM and is shown in Figure 4. The CHIT-g-PANI/ITO electrodes exhibited a relatively rough surface topology in comparison to CAH/CHIT-g-PANI/ITO electrode, which may facilitate the immobilization of $\mathrm{CAH}$ onto the CHIT- $g$-PANI/ITO electrode. To study the relative surface hydrophilicity, the contact angles of CHIT$g$-PANI/ITO and CAH/CHIT- $g$-PANI/ITO electrodes were measured [21] and were about $29 \pm 2^{\circ}$ and $38 \pm 2^{\circ}$, respectively. The work of adhesion between the surface of the electrodes and the water droplet $\left(W_{a}\right)$ can be calculated from the YoungDupre Equation (1):

$W_{a}=\gamma(1+\cos \theta)$

where $\gamma$ is the surface tension and $\theta$ is the contact angle. If the liquid is attracted to the solid surface (e.g., water on a strongly hydrophilic solid), the droplet will completely spread out on the solid surface and the contact angle will be close to $0^{\circ}$. According to this equation, the work of adhesion is higher at a lower contact angle. Since the contact angle increased after the CAH was immobilized on the surface of the CHIT- $g$-PANI/ITO electrode, this indicates that the CAH/CAH/CHIT- $g$-PANI/ ITO electrode surface has a weaker affinity to the hydrophilic moiety than CHIT- $g$-PANI/ITO electrode surface. 


\subsection{Electrochemical measurement}

Figure 5 shows the $\mathrm{CVs}$ of the electrochemical cells using either CHIT- $g$-PANI/ITO or $\mathrm{CAH} /$ CHIT- $g$-PANI/ITO electrode at a constant $50 \mathrm{mVs}^{-1}$ scan rate in $50 \mathrm{mM}$ phosphate buffer solution $(\mathrm{pH} 7.0,0.9 \% \mathrm{NaCl})$ containing $5 \mathrm{mM} \mathrm{Fe}(\mathrm{CN})_{6}^{3-14-}$. The current of the electrochemical cell using the electrode CHIT-g-PANI/ITO $\left(7.69 \cdot 10^{-6} \mathrm{~A}\right)$ was about five times of that using the CAH/CHIT- $g$ PANI/ITO bioelectrode $\left(1.72 \cdot 10^{-6} \mathrm{~A}\right)$.

Thus, immobilizing CAH onto the bare electrode reduced the current. A decrease in current after the immobilization of $\mathrm{CAH}$ may be attributed to a slower redox behavior when compared with the bare CHIT-g-PANI/ITO electrode. The covalent binding of CAH on the CHIT- $g$-PANI/ITO electrode controls the moment of the supporting electrolytes [11]. Also, the non-conducting nature of the $\mathrm{CAH}$ molecules might have contributed to the decrease in current when using the CAH/CHIT- $g$ PANI/ITO electrode.

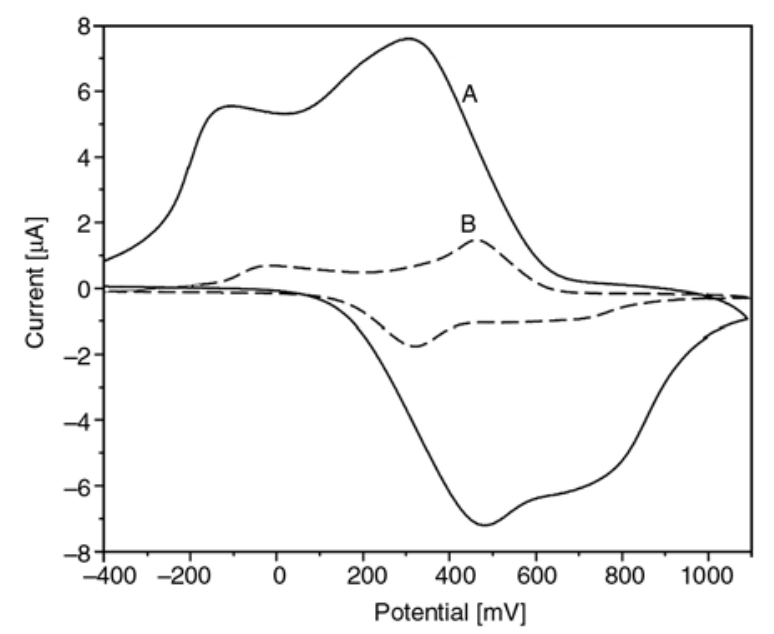

Figure 5. Cyclic voltammograms of the (A) CHIT- $g$-PANI/ ITO and (B) CAH/CHIT- $g$-PANI electrodes in PBS (50 mM, pH 7.0, 0.9\% NaCl, $5 \mathrm{mM}$ $\left.\mathrm{Fe}(\mathrm{CN})_{6}^{3-/ 4-}\right)$ at $50 \mathrm{mVs}^{-1}$ scan rate
The affinity of CAH to the CHIT- $g$-PANI/ITO graft copolymer matrix was estimated using the Hanes plot [22]. The Michaelis-Menten kinetic parameter $\left(K_{m}{ }^{a p p}\right)$ was calculated to be $0.51 \mathrm{mM}$ for the CAH/CHIT- $g$-PANI/ITO electrode. $K_{m}{ }^{a p p}$ usually depends on the electrode material as well as the enzyme immobilization process [23]. The $K_{m}{ }^{a p p}$ of the CAH/CHIT- $g$-PANI/ITO bioelectrode is much less than that of these previously reported biosensors (typically 2 to $7 \mathrm{mM}$ ). The small $K_{m}{ }^{a p p}$ value indicates a high affinity of CAH to the CHIT$g$-PANI matrix over the electrode surface, which may be attributed to 1) the advantageous $-\mathrm{NH}_{2}$ functionalized surface of the CHIT- $g$-PANI matrix for the enzyme immobilization that can favor conformational changes of the enzyme, and 2) the high positive electrostatic interaction, which can help to effectively immobilize CAH onto the CHIT-gPANI/ITO electrode.

\subsection{Photometric study}

A photometric study was performed to calculate the apparent enzyme activity of the $\mathrm{CAH}$ enzyme. $\mathrm{CAH}$ catalyzes the hydrolysis of creatinine (II) to produce sarcosine (III) and urea (IV). In the presence of urease, urea further hydrolyzed into ammonia, which in turn reacts with the Nessler's reagent $\left(\mathrm{K}_{2} \mathrm{Hg}_{2} \mathrm{I}_{4}\right)$ to form a colored product, $\mathrm{NH}_{2} \mathrm{Hg}_{2} \mathrm{I}_{3}$ (Figure 6). Through taking the absorbance of $\mathrm{NH}_{2} \mathrm{Hg}_{2} \mathrm{I}_{3}$ at $385 \mathrm{~nm}$, apparent enzyme activity of the CAH enzyme can be determined [24]. The apparent enzyme activity $\left({ }^{e n z} a_{a p p}\right)$ was calculated using Equation (2):

$$
{ }^{e n z} a_{\text {app }}=\frac{A V}{\varepsilon t s}
$$

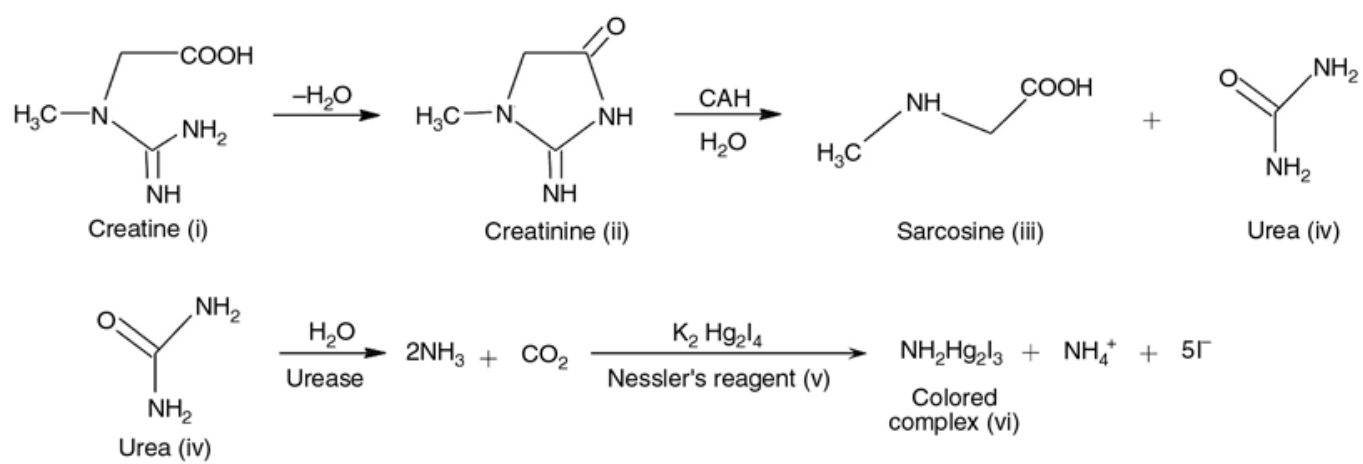

Figure 6. Production of photomerically active $\mathrm{NH}_{2} \mathrm{Hg}_{2} \mathrm{I}_{3}$ as a sensing element resulting from the reaction of Nessler's reagent and librated $\mathrm{NH}_{3}$ (i.e., obtained from enzymatic hydrolysis of creatinine) 
where $A$ is the difference in absorbance before and after incubation, $V$ is the total volume of the solution, $\varepsilon$ is the millimolar extinction coefficient, $t$ is the reaction time and $s$ is the surface area of the electrode. The apparent enzyme activity was calculated to be $83.59 \mathrm{mg} / \mathrm{cm}^{2}$; indicating $83.59 \mathrm{mg}$ of $\mathrm{CAH}$ was actively immobilized per unit area of CHIT- $g$-PANI matrix.

\subsection{Effect of $\mathrm{pH}$, temperature and time}

The peak current varied with the value of $\mathrm{pH}$ in the range 6.0-8.0; the optimum current is obtained at $\mathrm{pH}$ 7.0. It suggests that optimum CAH enzyme activity is found at $\mathrm{pH}$ 7.0. The thermal stability of the CAH/CHIT- $g$-PANI/ITO bioelectrode was studied by measuring the current at different temperatures ranging from 25 to $45^{\circ} \mathrm{C}$ in the presence of creatinine $150 \mu \mathrm{M}$ and a phosphate buffer (50 mM, pH 7.0). It was observed that the reaction rate increased with the temperature up to $32^{\circ} \mathrm{C}$ and the optimum temperature range was between 30 $32^{\circ} \mathrm{C}$ due to the increased kinetic energy of the reacting molecules. The storage stability of the CAH/CHIT- $g$-PANI/ITO electrode was amperometrically measured and a similar current response was found after it was stored for $\sim 300$ days at $4^{\circ} \mathrm{C}$. Hence, the CAH/CHIT- $g$-PANI/ITO bioelectrode exhibited an excellent operational and storage stability.

\section{Conclusions}

Creatine amidinohydrolase was covalently immobilized onto the CHIT-g-PANI graft copolymer matrix. The relatively low Michaelis-Menten constant of $0.51 \mathrm{mM}$ indicates that the CHIT- $g$-PANI matrix had a high affinity for the CAH enzyme. The enzyme holding capacity of graft copolymer matrix was determined to be $83.59 \mathrm{mg} / \mathrm{cm}^{2}$ and was affected by $\mathrm{pH}$, temperature, and time. Present efforts aim to use CAH/CHIT- $g$-PANI/ITO bioelectrodes with a good shelf life to fabricate an efficient creatinine biosensor that can detect creatinine from the blood and urine.

\section{Acknowledgements}

The authors are thankful to the Department of Science and Technology, Govt. of India for generous financial support and to the Director, National Physical Laboratory, New Delhi, India for providing infrastructure facilities to carry out this work.

\section{References}

[1] Burtis C. A., Ashwood E. R.: Tietz textbook of clinical chemistry. Saunders, Philadelphia (1986).

[2] Sena S. F., Syed D., McComb R. B.: Effect of high creatine content on the Kodak single-slide method for creatinine. Clinical Chemistry, 34, 594-595 (1988).

[3] Tsuchida T., Yoda K.: Multi-enzyme membrane electrodes for determination of creatinine and creatine in serum. Clinical Chemistry, 29, 51-55 (1983).

[4] Winquist F., Lundstrom I.: Determination of creatinine by an ammonia-sensitive semiconductor structure and immobilized enzymes. Analytical Chemistry, 58, 145-148 (1986). DOI: $\underline{10.1021 / \mathrm{ac} 00292 \mathrm{a} 035}$

[5] Yamato H., Ohwa M., Wernet W.: A polypyrrole/ three-enzyme electrode for creatinine detection. Analytical Chemistry, 67, 2776-2780 (1995). DOI: $10.1021 / \mathrm{ac} 00113 \mathrm{a} 009$

[6] Koncki R.: Recent developments in potentiometric biosensors for biomedical analysis. Analytica Chimica Acta, 599, 7-15 (2007). DOI: 10.1016/j.aca.2007.08.003

[7] Koncki R., Walcerz I., Ruckruh F., Glab S.: Bienzymatic potentiometric electrodes for creatine and Larginine determination. Analytica Chimica Acta, 333, 215-222 (1996). DOI: $\underline{10.1016 / 0003-2670(96) 00266-8}$

[8] Kubo I., Karube I., Suzuki S.: Amperometric determination of creatinine with a biosensor based on immobilized creatininase and nitrifying bacteria. Analytica Chimica Acta, 151, 371-376 (1983). DOI: $\underline{10.1016 / \mathrm{S} 0003-2670(00) 80098-7}$

[9] Schumann J., Möllering H., Jaenicke R.: Intrinsic stability and extrinsic stabilization of creatinase from Pseudomonas putida. Biological Chemistry HoppevSeyler, 374, 427-434 (1993).

[10] Schumann J., Böhm G., Jaenicke R., Schumacher G., Rudolph R.: Stabilization of creatinase from Pseudomonas putida by random mutagenesis. Protein Science, 2, 1612-1620 (1993). DOI: $10.1002 /$ pro. 5560021007

[11] Tiwari A., Gong S.: Electrochemical study of chitosan- $\mathrm{SiO}_{2}-\mathrm{MWNT}$ composite electrodes for the fabrication of cholesterol biosensors. Electroanalysis, 20, 2119-2126 (2008). DOI: $\underline{10.1002 / \text { elan.200804296 }}$ 
[12] Berberich J. A., Yang L. W., Bahar I., Russell A. J.: A stable three enzyme creatinine biosensor. 2. Analysis of the impact of silver ions on creatine amidinohydrolase. Acta Biomaterialia, 1, 183-191 (2005).

DOI: $10.1016 /$ j.actbio.2004.11.007

[13] Tiwari A.: Gum arabic-graft-polyaniline: An electrically active redox biomaterial for sensor applications. Journal of Macromolecular Science Part A: Pure and Applied Chemistry, 44, 735-745 (2007).

DOI: $10.1080 / 10601320701353116$

[14] Tiwari A., Gong S.: Electrochemical detection of a breast cancer susceptible gene using cDNA immobilized chitosan-co-polyaniline electrode. Talanta, 77, 1217-1222 (2009).

DOI: $\underline{10.1016 / j . t a l a n t a .2008 .08 .029}$

[15] Tiwari A., Gong S.: Electrochemical synthesis of chitosan-co-polyaniline/ $\mathrm{WO}_{3} \cdot n \mathrm{H}_{2} \mathrm{O}$ composite electrode for amperometric detection of $\mathrm{NO}_{2}$ gas. Electroanalysis, 20, 1775-1781 (2008).

DOI: $\underline{10.1002 / \text { elan.200804237 }}$

[16] Tiwari A., Singh V.: Synthesis and characterization of electrical conducting chitosan-graft-polyaniline. Express Polymer Letters, 1, 308-317 (2007). DOI: $10.3144 /$ expresspolymlett.2007.44

[17] Tiwari A., Sen V., Dhakate S. R., Mishra A. P., Singh V.: Synthesis, characterization and hoping transport properties of $\mathrm{HCl}$ doped conducting biopolymer-copolyaniline zwitterion hybrids. Polymers for Advanced Technology, 19, 909-914 (2008). DOI: $10.1002 /$ pat. 1058

[18] Tiwari A., Singh V.: Microwave-induced synthesis of electrical conducting gum acacia-graft-polyaniline. Carbohydrate Polymers, 74, 427-434 (2008).

DOI: 10.1016/j.carbpol.2008.03.015
[19] Tiwari A.: Synthesis and characterization of $\mathrm{pH}$ switching electrical conducting biopolymer hybrids for sensor applications. Journal of Polymer Research, 15, 337-342, (2008).

DOI: $10.1007 / \mathrm{s} 10965-008-9176-4$

[20] Solanki P. R., Kausik A. K., Ahamad A. A., Tiwari A., Malhotra B. D.: Multi-walled carbon nanotubes/solgel derived silica/chitosan nanobiocomposite for total cholesterol sensor. Sensors and Actuators B: Chemical, 137, 727-735 (2009).

DOI: $10.1016 /$ j.snb.2008.12.044

[21] Abbasian A., Ghaffarian S. R., Mohammadi N., Fallahi D.: The contact angle of thin-uncured epoxy films: Thickness effect. Colloids and Surfaces A: Physicochemical and Engineering Aspects, 236, 133 140 (2004). DOI: $10.1016 /$ j.colsurfa.2004.01.028

[22] Wilson K., Walker J. M.: Principles, techniques of practical biochemistry. Cambridge University Press, New York (2000).

[23] Kouassi G. K., Irudayaraj J., McCarty G.: Examination of cholesterol oxidase attachment to magnetic nanoparticles. Journal of Nanobiotechnology, 3, 1-9 (2005).

DOI: $10.1186 / 1477-3155-3-1$

[24] Rajesh, Bisht V., Takashima W., Kaneto K.: An amperometric urea biosensor based on covalent immobilization of urease onto an electrochemically prepared copolymer poly ( $N$-3-aminopropyl pyrrole-copyrrole) film. Biomaterials, 26, 3683-3690 (2005). DOI: 10.1016/j.biomaterials.2004.09.024 